\title{
DESCARTES EN AFRIQUE
}

\author{
Simha Arom (Lacito-CNRS, Paris) \\ Jean K'lalfa (Trinity College, Cambridge, Royaume-Uni)
}

\section{Les musiques traditionnelles, domaine privilégié d'étude de la rationalité}

S'il est un domaine où l'on s'attendait peu à trouver la raison pure en acte, c'est bien celui des musiques traditionnelles africaines, souvent perçues en Occident comme expression de la spontanéité et de l'improvisation, sinon du chaos. Impression qu'explique entre autre le caractére insaisissable de musiques transmises par la seule voie orale, et exécutées à des tempi défiant toute analyse qui ne s'aiderait d'enregistrements et de transcriptions. Or les recherches récentes sur ces musiques révèlent l'étonnante maitrise de structures complexes qui sous-tend leur exécution et leur comprélension (Arom 1985).

Où l'on voit que le bon sens musical est la chose d'une culture la mieux partagée...

L'organisation de la musique dans les société traditionnelles d'Afrique Centrale peut s'analyser à deux niveaux: son articulation avec le contexte socioculturel et son fonctionnement intrinsèque comme système sémiotique.

En général, dans chaque groupe culturel, toute pièce musicale possède un titre et correspond à une fonction, elle-mème liée à une ou plusieurs circonstances particulières. Liensemble des pièces spécifiques à cette fonction constitue un répertoire. Ainsi la naissance de jumeaux donne-t-elle lieu à un rituel comprenant un nombre donné de chants accompagnés d'une même formation instrumentale, composée le plus souvent d'instruments percussifs et partageant un même soubassement polyrythmique.

Chaque répertoire constitue une catégorie musicale, dotée elle aussi d'un nom en langue vernaculaire, généralement celui de la circonstance de son exécution. Dans une communauté ethnique donnée, il n'est pas de pièce musicale qui n'appartienne à une catégorie. Lorsqu'une circonstance se satisfait d'une seule pièce, celle-ci porte le nom de la circonstance en question, mais elle se distingue de surcroît de toutes les autres pièces du patrimoine musical par un ou plusieurs traits strictement musicaux.

Ainsi, chez les Pygmées Aka, I'interdiction de tout accompagnement du chant de déploration sur le cadavre, Koli (qui signifie «mort»), l'opposent aux autres catégories musicales de ce groupe, dont toutes les pièces peuvent être accompagnées.

Enfin, les catégories s'excluent - une mème pièce ne peut figurer dans deux catégorieset le nombre des catégories est fini. La taxonomie musicale vernaculaire coïncide donc avec l'organisation cognitive du patrimoine musical: une catt́gorie se définit non seulement par des traits socioculturels mais aussi par des traits structurels complexes, articults d'une façon rigoureusement systématique et -à l'intérieur d'une mème culture- universelle: chaque individu du groupe possède intuitivement les axiomes du système et participe à sa mise en oeuvre. Cette rationalite de type cartesien se retrouve dans pratiquement toute l'Afrique subsaharienne.

Dans les sociétés traditionnelles de cette région, la transmission du savoir est orale, et toute connaissance est mémoire, mémoire "collective» qui se forme par observation, imitation et, dans certains cas, initiation. A cette exception près, il n'y a pas d'apprentissage institutionnalisé, mais on encourage les enfants, en tout temps et en tout lieu, à reproduire ce qu'ils observent et à participer à la vie sociale du groupe. L'organisation de la musique n'en relève pas moins de principes mathématiques très complexes. axiomes dont les dépositaires 
ne sont pas conscients, sauf à parler d'un cadre structurel de la conscience musicale, consistant précisément en l'emploi et le respect d'un ensemble de règles, c'est-à-dire d'une théorie implicite ou "en creux», ne se réfléchissant elle-méme que lorsque se produisent un écart ou une erreur.

Dans ces sociétés, l'analyse de l'organisation de la musique dans le temps, c'est à dire des structures rythmiques et de la trame métrique sur laquelle elles se déploient, révèle une immense complexité, égalée dans aucune autre musique de tradition orale.

\section{Principes de structuration du temps musical}

En Afrique Centrale, n'est considérée comme relevant de la musique qu'une séquence sonore dont toutes les durées entretiennent des rapports strictement proportionnels. Dans chaque pièce, ces rapports ont pour commun dénominateur une durée métronomique constante, prise comme étalon. Aussi bien n'y considère-t-on comme musique que ce qui se prête à la danse: la synchronisation des pas des danseurs nécessite en effet un étalonnage du tenips.

Or la structuration des durées de toute séquence musicale relève d'une organisation mathématique fondée sur des principes concernant, d'une part, le cadre périodique dans lequel les événements sonores se produisent —ordre métrique - et, d'autre part, leur articulation au sein de ce cadre —ordre rythmique. La métrique concerne l'étalonnage du temps en quantités -ou valcurs - égales; le rythme, les modalités de groupement de ces valeurs. La métrique est un contimum, un fond ou une trame muette sur laquelle se déploie cette forme temporelle qu'est le rythme (Arom 1992 a).

Les principes métriques sont les suivants: toute musique est composée de cycles périodiques fondés sur des pulsations régulières - matérialisées ou non par un son. Ces pulsations sont toutes de statut équivalent, sans accentuation aucune lorsqu'elles sont audibles. C'est pourq̨uoi, entre la période prise comme un tout et la pulsation, il n'y a pas ici place pour la "mesure», convention graphique utilisée par la musique occidentale depuis le 17 e siècle pour désigner une quantité constante de valeurs dont le réitération est marquée par un "temps fort».

Toute entité musicale —qu'il s'agisse d'une séquence monodique ou d'une polyphonie ou polyrythmie complexe- est donc tributaire d'un cadre périodique fixe, fondé sur un nombre entier —et généralement pair- de pulsations régulières, équidistantes, qui en constituent l'armature métrique. La pulsation se subdivise, selon les cas, en deux, trois, ou parfois même cing valeurs opérationnelles minimales. La valeur opérationnelle minimale équivaut à la plus petite durée pertinente issue de ce monnayage et toutes les autres durées en constituent nécessairement des multiples. C'est à partir de ces valeurs minimales que s'élabore la contiguration rythmique propre à chaque pièce et, dans la polyphonie ou la polyrythmie, à claque partic.

Bien qu'en principe, dans les musiques traditionnelles d'Afrique Centrale, la période soit toujours divisible de façon symétrique, son articulation ryl/mique présente le plus souvent une asymétrie interne; en d'autres termes, la symétrie de l'organisation inétrique est constamment rompue par l'ordonnancement de la substance rythmique qui vient se greffer sur elle. Ces ruptures de symétrie procèdent toutes d'un même principe, celui de contramétricité (Arom 1992 b). Larticulation du rythme est contramétrique lorsque les accents, ou -en leur absence- les attaques des sons, interviennent pour l'essentiel à contretemps, et ce de façon irrégulière, puisqu'une asymétrie rythmique qui serait régulièrement décalèe par rapport à la pulsation pourrait être perçue comme symétrique par rapport à un cadre métrique différent.

Les manifestations les plus remarquables de la contramétricité sont l'/émiole et l'imparitè rythmique. 
Par hémiole, il faut entendre qu'entre le nombre de pulsations d'un cycle périodique et le contenu d'une figure rythmique prévaut un rapport de $2: 3$, de 3:4, ou de l'un de leurs multiples (4:6 ou $6: 8$ par exemple).

L'hémiole n'est autre que la répétition, au sein d'une période, d'une même figure rythmique mais dont la position par rapport à la pulsation se trouve décalée à chaque itération. jusqu'à ce qu'elle coïncide à nouveau avec la pulsation, ce qui marque le début de la période suivante. Ce décalage résulte de la superposition de deux progressions arithmétiques de raisons différentes, l'une métrique, l'autre rythmique. Dans la musique occidentale, leur rapport est le plus souvent $3: 2$, alors qu'en Afrique le rapport 4:3 est tout aussi fréquent.

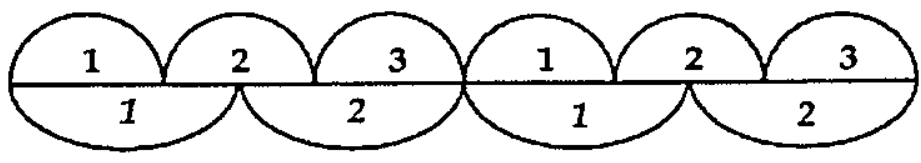

EXEMPLE 1

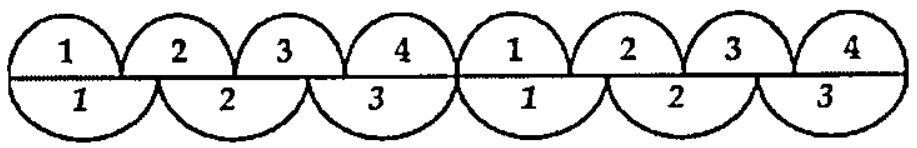

\section{EXEMPLE 2}

On voit bien que dans un cas comme dans l'autre la période ne recommence que lorsque le son initial de la figure rytlımique coïncide avec la pulsation, soit après deux ou trois itérations.'

L'imparité n'thmique est un procédé extrêmement subtil, qui semble spécifique da la musique africaine. Comme on l'a déjà vu, le résultat de la division en deux du nombre total de valeurs opérationnelles minimales d'une période est un nombre pair. Or, l'agencement des tigures qui relevent de l'imparité rythmique est tel que toute tentative de les segmenter au plus près du point de division central de la période résultera inévitablement en deux parties inégales, constituées chacune d'un nombre de valeurs minimales impair -et ce, quel que soit. au sein de la figure, le point de départ que l'on se donne. Elles se présentent toujours sous la lorme de deux blocs, dont la distribution correspond, sur la base des valeurs minimales, à moitie $+1 /$ moitié -1 . L'articulation de ces figures résulte de la juxtaposition de groupements inégaux de quantités binaires et temaires.

L'exemple qui suit présente en a) les percussions d'un instrument de rythme, en b) les quatre pulsations, en c) les douze valeurs minimales. On voit que l'articulation de la ligure rythmique ne coïncide pas avec l’organisation métrique de la période. Elle la contredit.

\begin{tabular}{|c|c|c|c|c|c|c|c|c|c|}
\hline a) & $\theta$ & & 10 & 10 & $\otimes$ & 1 & & 0 & \\
\hline b) & & & & & 1 & & & & \\
\hline c) & 1 & 2 & 3 & 6 & 7 & 9 & 10 & 11 & 12 \\
\hline
\end{tabular}

\section{EXEMPLE 3}

I Notons que lat période globale de tout clant qui aurait pour accompagnament l'ttne de ces deux tigures rihmiques sera nécessairement un multiple entier de la période qu'elles déterminant. 
L'articulation de cette figure rythmique ne coïncide jamais avec un division de la période en deux parties égales (6/6); tout au plus peut-on tenter d'aller au plus près du point de division central. Soit une suite d'éléments $\left(3 \cdot 2 \cdot 2 \cdot 3 \cdot 2 \cdot 3 \cdot 2 \cdot 2\right.$, etc. $\left.{ }^{2}\right)$ : quel que soit l'événement rythmique $f$ à partir duquel le comptage commence, jamais l'un n'obtiendra deux blocs de 6 dans une periode comptant 12 valeurs opérationnelles minimales, mais toujours 5 et 7 . par $(3 \cdot 2) /(3 \cdot 2 \cdot 2)$, ou bien 7 et $5, \operatorname{par}(3 \cdot 2 \cdot 2) /(3 \cdot 2)$.

Dans les exemples qui suivent, chaque cercle représente une période. Dans le premier cas (Exemple 4), cette période englobe douze valeurs minimales, correspondant à 4 pulsations. II s'agit de la même figure rythmique que dans l'exemple 3 , à savoir $(3 \cdot 2 \cdot 2 \cdot 3 \cdot 2)$.

\section{EXEMPLE 4}

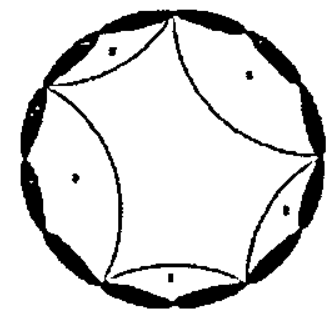

Dans l'exemple suivant, la période comprend aussi 4 pulsations, mais qui correspondent cette fois à 16 valeurs minimales - la subdivision des temps est donc binaire; la période inclut la suite rythmique $(3 \cdot 2 \cdot 2 \cdot 2 \cdot 3 \cdot 2 \cdot 2)$. Là encore, il est impossible de scinder cette suite en deux parties égales ; en effet, quel que soit le point de départ choisi au sein du cycle, l'on obtiendra tou-jours 9 et $7, \operatorname{par}(3 \cdot 2 \cdot 2 \cdot 2) /(3 \cdot 2 \cdot 2)$ ou bien 7 et 9 , $\operatorname{par}(3 \cdot 2 \cdot 2) /(3 \cdot 2 \cdot 2 \cdot 2)$.

\section{EXEMPLE 5}

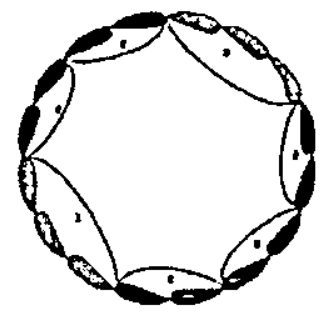

Élargissons maintenant la période. Dans l'exemple qui suit, le cycle totalise huit pulsations qui se subdivisent de fą̧on ternaire, soit en tout 24 valeurs mi-nimales $(3 \cdot 2 \cdot 2 \cdot 2 \cdot 2$. $2 \cdot 3 \cdot 2 \cdot 2 \cdot 2 \cdot 2$ ). Là encore, en partant d'un quelconque élément, il est impossible de segmenter la figure en deux parties égales. Quel que soit le point de départ, l'on aboutira toujours à deux blocs impairs, l'un regroupant 13 valeurs minimales, l'autre 1 I.

EXEMPLE 6

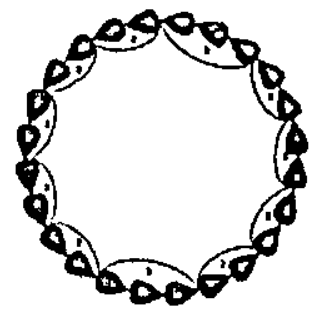

2 Le symbole $\cdot$ désigne la concaténation des groupements de valeurs minimales opérationnelles. 
Le principe de l'imparité rythmique peut être appliqué d des périodes de dimensions différentes, selon un procédé d'augmentation qui consiste à insérer des groupements binaires dans des configurations délimitées par un groupement ternaire. Ce que résume le tableau suivant:

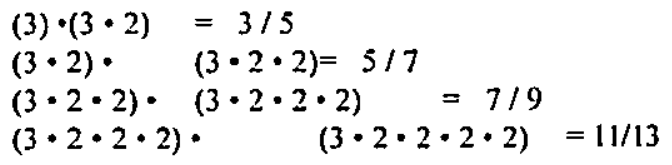

\section{EXEMPLE 7}

Les diffërents types de contramétricité que sont l'hémiole et l'imparité rythmique montrent particulièrement bien que toute figure rythmique est indissociable d'une organisation métrique spécifique qui la constitue comme unité de perception mais qui, le plus souvent, demeure littéralement sous-entendue. Or, en fonction de la position de la pulsation, toute figure rythmique est en principe compatible avec plusieurs organisations métriques. Lorsque le nombre total de valeurs opérationnelles minimales d'une période n'est divisible que par 2 et par 4 (8 ou 16 par exemple), il y a quatre possibilités. Lorsqu'il est divisible par 4 et par 3 ( 12 ou 24 par exemple), trois autres possibilites viennent s'y ajouter. La projection de chacune des 4 ou 7 organisations métriques possibles sur une même séquence musicale engendre une perception à chaque fois radicalement différente de cette séquence (le lecteur peut en faire l'expérience à l'aide d'un métronome). Il faut donc bien supposer que tous les participants à un événement musical —c'est à dire. dans ces sociétés, l'ensemble de la communautí-, se réfêrent mentalement à une seule et méme trame métrique. Autrement dit, ce qui s'entend n'a de sens que dans un rapport complexe à une substance, au sens cartésien, qui seule peut en rendre raison.

\section{DE LA MÉTHODE...}

A l'écoute de la plupart des musiques traditionnelles d'Afrique Centrale se dígage une impression d'incertitude. L'auditeur non-initié a peine à y déceler des points de repère temporels, même si le retour d'événements musicaux semblables à intervalles réguliers l'incite à chercher, mais en vain, une organisation métrique régulière: rien ne lui permet a priori de déterminer l'étalon auquel ces durées se rétêrent. L'incertitude nait du contlit permanent qui oppose, à la régularité induite par les différents cadres périodiques possibles, l'articulation asymétrique du matériau rylhmique qui y est inséré.

Mais le chercheur confronté à ce matériau peut, par l'observation et l'expérimentation, vérifjer l'existence d'un référent que l'on pourrait qualifier de transcendantal.

Dans les danses collectives, les pas de base communs à tous les danseurs sont toujours isochrones. Il est done raisonnable de postuler qu'une telle isochronie coïncide avec la pulsation qui détermine l'organisation métrique de la pièce. Mais, comme on ne peut exclure a priori que tous les danseurs soient à contretemps, il faut tester cette hypolliese. On peut le faire aisément en demandant à un membre de la communauté de superposer des battements de main à un enregistrement de la síquence qu"on lui fait enlendre. Malgré l'extrnordinaire complexité rythmique de cette musique, et à de rarissimes exceptions près, il matérialisera instantanément la pulsation inhérente à cette séquence, dévoilant par là nềme ses modalités de division en valeurs opérationnelles binaires ou ternaires. 
C'est alors, et alors seulement, que le chercheur pourra considérer à bon droit détenir une clef d'accès à l'organisation mentale qui sous-tend une musique donnée. L'expérience pourra être répétée à des intervalles de plusieurs mois, voire plusicurs années, et auprès de quelque membre de la communauté que ce soit, le résultat sera toujours le mẻme.

Une fois découvert, le cadre métrique joue un rôle essentiel dans la vérification de la validité de la transcription d'une pièce. Car une transcription qui produirait des figures rythmiques dont la somme des valeurs minimales ne serait pas égale au total des valeurs minimales de la période qu'il aurait préalablement déterminées, serait nécessairement inexacte. L'identification de la pulsation inhérente à chaque pièce rend donc enfin possible la transcription et l'analyse de ces musiques, des plus simples aux plus complexes, dans le respect de la conception de /'organisation temporelle qui les sous-lend: Loin de n'être qu'une des multiples interprétations subjectives également compatibles avec le matériau sonore, une telle conception est en fait un principe formel d'organisation, structurant l'univers musical d'un groupe donné. C'est ce que confirme la taxonomie vernaculaire. Ainsi ne peut-on guère comprendre, chez les Gbaya de Centrafrique, le principe de division du répertoire des "chants à penser» en deux «familles», si l'on ne considère que leur contenu littéraire. L'analyse musicologique révèle que, si les deux familles sont fondées sur une même périodicité ( 8 pulsations par période), dans l'une chaque pulsation fait l'objet d'une subdivision ternaire, alors que dans l'autre cette subdivision est binaire. De ce fait tous les chants de la première famille comptent 24 valeurs minimales, alors que ceux de l'autre en comptent toujours 32. En identifiant des traits musicaux structurels, une telle analyse corrobore donc la classification des Gbaya (Dehoux 1986). Et l'on pourrait inultiplier les exemples de ce type.

Dans des sociétés où la théorie musicale est implicite, il s'avère que la pratique s'appuie néanmoins sur des principes mathématiques stricts permettant d'engendrer et de maitriser des structures hautement complexes. Chez les musiciens traditionnels africains, la mathesis aussi est universelle, mème si, comme le dit Leibniz, "L'âme calcule mais ne le sait pas».

\section{RÉFÉRENCES BIBLIOGRAPHIQUES}

AROM, SIMHA: Polyphonies et Polyrythmies instrumentales d'Afrique Centrale. Siructure et méthodologie. Paris: SELAF (Ethnomusicologie 1), 2 vol, 1985.

$-A$ la recherche du "tentps " perdu: métrique el rythme en musique, in J.-J. Wunen-burger (éd.), Les rythmes. Lectures et théories. Actes du Colloque sur le rythme. Cerisy-la-Salle 1989. Paris: L'Harmattan, p. 195-205. 1992.

-Symétrie et ruptures de symétrie dans les musiques de tradition orale: le cas de l'Afrique Centrale, Quadrivium. Alusiques et Sciences. Paris: IPMC La Villette, p. 209-216. 1992.

- (Intelligence in Traditional Music», in J. Khalfa (ed.), What is Intelligence? Danvin College Lectures 1992. Cambridge and New York: Cambridge Univer-sity Press, p. 137-160. 1992. DEHOUX, VINCENT: Chants à penser Gbaya (Centrafrigue). Paris: SELAF (Ethnomusicologie 2).1986. 